\title{
Evaluating Musical Foreshadowing of Videogame Narrative Experiences
}

\author{
Marco Scirea \\ IT University of Copenhagen \\ Rued Langgaards Vej 7 \\ DK-2300 Copenhagen S \\ marco.prolog@gmail.com \\ Mark J. Nelson \\ IT University of Copenhagen \\ Rued Langgaards Vej 7 \\ DK-2300 Copenhagen S \\ mjas@itu.dk
}

\author{
Yun-Gyung Cheong \\ IT University of Copenhagen \\ Rued Langgaards Vej 7 \\ DK-2300 Copenhagen S \\ yugc@itu.dk \\ Byung-Chull Bae \\ Sungkyunkwan University \\ 25-2 Sungkyunkwan-Ro \\ Seoul, South Korea \\ byuc@skku.edu
}

\begin{abstract}
We experiment with mood-expressing, procedurally generated music for narrative foreshadowing in videogames, investigating the relationship between music and the player's experience of narrative events in a game. We designed and conducted a user study in which the game's music expresses true foreshadowing in some trials (e.g. foreboding music before a negative event) and false foreshadowing in others (e.g. happy music that does not lead to a positive event). We observed players playing the game, recorded analytics data, and had them complete a survey upon completion of the gameplay. Thirty undergraduate and graduate students participated in the study. Statistical analyses suggest that the use of musical cues for narrative foreshadowing induces a better perceived consistency between music and game narrative. Surprisingly, false foreshadowing was found to enhance the player's enjoyment.
\end{abstract}

\section{Categories and Subject Descriptors}

H.5.5 [Information Interfaces and Presentation]: Sound and Music Computing

\section{General Terms}

Experimentation

\section{Keywords}

Music generation, Narrative, Procedurally generated content

\section{INTRODUCTION}

In games, unlike in traditional linear storytelling media such as novels or movies, narrative events unfold in response

Permission to make digital or hard copies of all or part of this work for personal or classroom use is granted without fee provided that copies are not made or distributed for profit or commercial advantage and that copies bear this notice and the full citation on the first page. Copyrights for components of this work owned by others than ACM must be honored. Abstracting with credit is permitted. To copy otherwise, or republish, to post on servers or to redistribute to lists, requires prior specific permission and/or a fee. Request permissions from Permissions@acm.org.

AM '14, October 01 - 03 2014, Aalborg, Denmark Copyright 2014 ACM 978-1-4503-3032-9/14/10\$15.00.

http://dx.doi.org/10.1145/2636879.2636889 to the player input. Therefore, the music composer in an interactive environment needs to create music that is dynamic and non-repetitive. Dynamic music creation is not new. Algorithmic music composition has been actively researched for the last several decades with a large variety of approaches. Examples are Mezzo [8] (that focuses on creating Renaissance style music through manipulation of leitmotifs), the Cell-based approach [16] (used in Tom Clancy's EndWar) and using neural networks to create musical improvisations [37].

The use of music for foreshadowing has been better studied in film (see e.g. Cohen [10]). The soundtrack keeps the viewer engaged in the story [14], particularly in the horror movie genre, in which music foreshadows the upcoming events. Foreshadowing also impacts the recall of story events in film. Boltz et al. [6] conducted an experimental study which revealed that events preceded by false foreshadowing are better remembered by viewers.

There have also been several computational models of narrative generation that have tackled the topic of foreshadowing. An example is Minstrel [30], a case-based reasoning system which can decide what and when to foreshadow by considering the uncommonness and similarity of actions and states. Another example is the narrative generation system developed by Bae and Young [1], which uses Chekhov's Gun ${ }^{1}$ style foreshadowing.

Music-based foreshadowing in games can be often found in cutscenes where no player interaction occurs. In normal gameplay, audio cues via sound effects are fairly common (an interesting non-videogame example is the use of audio foreshadowing in a gambling machine [31]). However, there is little research (none, to our knowledge) about the use of music (rather than other kinds of audio) for foreshadowing in games. Therefore, we attempt to explore affective and

\footnotetext{
1 "Remove everything that has no relevance to the story. If you say in the first chapter that there is a rifle hanging on the wall, in the second or third chapter it absolutely must go off. If it's not going to be fired, it shouldn't be hanging there." Anton Chekhov
} 
cognitive modeling, coupled with real-time adjustment of the music.

The research questions we attempt to answer in this paper are:

1. In games, can procedurally generated, mood-expressive music serve as an indicator of narrative foreshadowing?

2. Can the use of musical foreshadowing improve the player's experience?

The moods that we deal with in this paper are bored, content, happy, miserable, tired, fearful, peaceful and alarmed; these are based on Russell's direct circular projection of adjectives [33] with some slight modifications.

To answer these questions we conducted an empirical evaluation and analyzed the collected user response data. Section 2 reviews existing theories on emotions, narrative foreshadowing and the state-of-the-art of procedurally generated music to then delineate our hypothesis (section 3 ). In section 3.2 we will discuss the various data collection methods we employed, while in section 4 we analyze the data to finally discuss the results in section 5 .

This paper does not discuss the details of our mood dependent music generator. We refer the reader to [34].

\section{BACKGROUND THEORIES}

In this section we briefly discuss the background theories upon which our approach is based.

\subsection{Procedurally Generated Music}

Procedural generation of music content is a booming field that has seen a tremendous growth lately, which can range from creating simple sound effects, to avoid repeating the same clip over and over, to entire game worlds [36]. While a good number of games use some sort of procedural music structure, there are different approaches (or degrees), as suggested by Wooller et al:: transformational algorithms and generative algorithms [40].

Transformational algorithms act upon an already prepared structure, for example by having the music recorded in layers that can be added or subtracted at a specific time to change the feel of the music (e.g., The Legend of Zelda: Ocarina of Time [29] is one of the earliest games that used this approach). Note that this is only an example and there are a great number of transformational approaches, but we won't discuss them in this paper.

Generative algorithms instead create the musical structure themselves, which leads to a higher degree of difficulty in having the music stay consistent with the game events and generally requires more computing power as the musical materials have to be created on the fly. An example of this approach can be found in Spore [28]: the music written by Brian Eno was created with Pure Data in the form of many small samples that created the soundtrack in real time.
Adopting the latter approach, we present generative procedural music generation in games for emotional expression. While the topics of affect [5], semiotics [12] and moodtagging [23] are also interesting and significant, our focus lies in the real-time generation of background music able to express moods.

\subsection{Emotions and Moods}

The topic of emotions has been extensively researched in the field of psychology, although what is their nature (or what are the basic ones) is still controversial. Lazarus argues that "emotion is often associated and considered reciprocally influential with mood, temperament, personality, disposition, and motivation" [19].

Affect is generally considered as the experience of feeling or emotion. It is largely believed that affect is post-cognitive; emotion arises only after an amount of cognitive processing of information has been accomplished. With this assumption every affective reaction (e.g., pleasure, displeasure, liking, disliking) results from "a prior cognitive process that makes a variety of content discriminations and identifies features, examines them to find value, and weighs them according to their contributions" (Brewin, [7]). Another view is that affect can be both pre and post-cognitive (notably Lerner and Keltner [20]); thoughts are being created by an initial emotional response which then lead to producing affect.

Mood is an affective state. However, while an emotion generally has a specific object of focus, moods tends to be more unfocused and diffused [27]. Batson, Shaw, and Oleson say that mood "involves tone and intensity and a structured set of beliefs about general expectations of a future experience of pleasure or pain, or of positive or negative affect in the future" [3]. Another important difference between emotions and moods is that moods, being diffused and unfocused, can last much longer (as also remarked by Beedie et al. [4]).

In this paper, we focus on moods instead of emotions, for we expect that in games-where the player listens to the background music for a longer time duration than the duration that a particular emotion is experienced- moods are more likely to be remembered by the players after their gameplays.

\subsection{Music mood taxonomy}

The set of adjectives that describe music mood and emotional response is immense and there is no accepted standard. For example in the work by Katayose et al.[17], the emotional adjective set includes Gloomy, Serious, Pathetic and Urbane.

Russell [33] proposed a model of affect based on two bipolar dimensions: pleasant-unpleasant and arousal-sleepy, theorizing that each affect word can be mapped into this bidimensional space by a combination of these two components. Thayer [39] applied Russell's model to music using as the dimensions of stress and arousal; although the names of the dimensions are different from Russell's their meaning is the same. Also, we find different names in different research while the semantic meanings are identical. We will use the terms valence and arousal, as they are the most commonly used affective computing research. Then the affect in music 
can be divided into the four clusters based on the dimensions of valence and arousal: Anxious/Frantic (Low Valence, High Arousal), Depression (Low Valence, Low Arousal), Contentment (High Valence, Low Arousal) and Exuberance (High Valence, High Arousal). These four clusters have the advantage of being explicit and discriminable; also they are the basic music-induced moods [18],[22].

\subsection{Foreshadowing}

Foreshadowing is a narrative technique where an uncertain, significant event is anticipated, being cued by a less important event. Chatman defines foreshadowing as the seeding of an anticipatory satellite from which a kernel can be inferred [9]. Chatman defines kernel as a key event in the story that advances the storyline and cannot be eliminated without harming the story, while satellite events are minor plot nodes that do not contribute to the main story line. He further suggests that foreshadowing leads to invoking suspense in the reader and surprise in the story character by creating a disparity in knowledge between the character and the reader (that is, the reader knows more information about uncertain future events).

Genette characterizes foreshadowing as advance mention, being an indirect and implicit reference that may or may not be confirmed in its importance [13]. He also makes a distinction between two types of advance mention: genuine and false advance mentions. Genuine advance mentions give the reader correct cues about the development of the story, while false advance mentions give misleading knowledge (also called snares by Barthes [2]). By mixing these two types of foreshadowing we can engage the reader in trying to analyze the clues and find out which are the true and false ones.

Music can be used to express foreshadowing. This can range from subtly playing in the background music the theme associated with one character to prepare for an entrance (e.g., the Indiana Jones franchise [24] makes great use of this expedient) to using the entire music at the time to foreshadow the next event (the shower scene in Hitchcock's Psycho [15]). As evidenced in these films, a great amount of degrees of emotional intensity (and suspense) can be expressed through foreshadowing: for example the latter example creates a much greater feeling of suspense that the former.

\section{METHODS}

This section describes our experiment and the data collection methods we employed.

\subsection{Experiment Design}

\subsubsection{Test groups}

To test the relationship between music and gameplay, we first designed three different test groups. Each participant plays a game that has background music that foreshadows the correct future mood (true foreshadowing), a wrong mood (false foreshadowing), and a basedline mood (static - "content" mood).

- Group 0: true foreshadowing.

- Group 1: false foreshadowing.
- Group 2: control group.

\subsubsection{Conditions}

The test was conducted in a relatively quiet and calm environment. Before the experiment started, the participants were given information about the game controls (mouse and keyboard for interactions), and were told how the mood selector works. Then, the participants played the game with some good quality noise-cancelling headphones. In general, it took about ten minutes to finish the game. When the game ended, the participants were asked to complete the survey.

\subsubsection{The Game}

To express foreshadowing the game was designed to have narrative component. Classically, narrative-heavy games have been adventure games, defined by Rollins et al. as "a video game in which the player takes the role of the protagonist in an interactive narrative driven by puzzle-solving an exploration" [32]. This genre had its peak of popularity in the early to mid 1990s with classical games like The Secret of Monkey Island[25] and Grim Fandango[26]. Lately it seems to reacquire success with the critically acclaimed The Walking Dead[38] and Broken Age[11].

This genre suits our needs very well, as its main focus is on storytelling, and the set of features to implement is relatively small compared to other genres, minimizing the need to expend develop effort on narratively extraneous game mechanics. Adventure games generally contains branching narratives, but our test game narrative progresses in a linear fashion, due to development time constraint.

The main gameplay consists of:

- Exploring the environment

- Picking up items (and generally interacting with them)

- Using items on the environment to solve puzzles

These gameplay actions are the most basic mechanics used in adventure games.

\subsubsection{Narrative/Emotional Design}

In this study, we investigated the following eight basic moods generated by the music generator [35]. The main moods that we identified in the story (in narrative chronological order) are:

- Miserable: in the beginning of the game, the girl who is the protagonist in the game is sad. This is the main mood.

- Happy/Content/Peaceful cluster: soon, it starts snowing and her mood becomes more positive. Foreshadowing can be applied in contrast with the previous mood.

- Alarmed: When the snow starts melting this is the main mood; we can use foreshadowing to give a musical cue to the ensuing drama. 

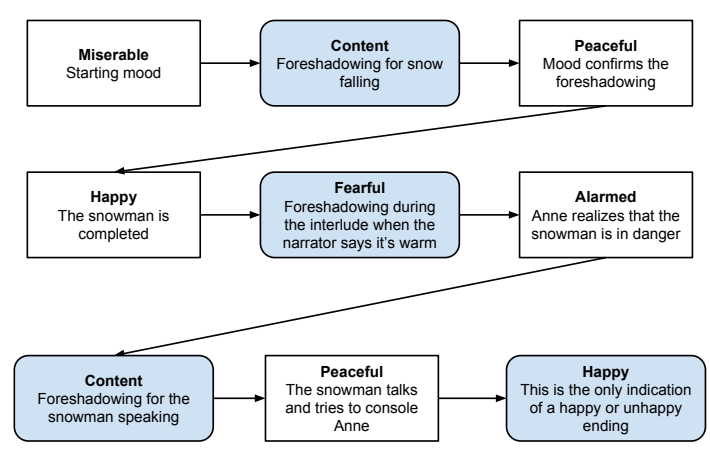

Figure 1: The emotional structure with true foreshadowing (for Group 0). In white we have the persistent moods and in blue the true foreshadowing ones.

- Peaceful: Resulting mood of the cathartic moment when the snowman speaks.

With this storyline associated with a particular mood, we identified three key story moments where we can use foreshadowing in a congruous and incongruous way (see figures 1 and 2).

The first significant event is when the snow starts falling, where the main character is uncertain whether this event is positive or negative. However, the resolution makes this positive, transiting the mood of the character to peaceful. Therefore, we foreshadow it with content for true foreshadowing and alarmed for false foreshadowing.

The second key event is the realization that the Snowman is going to melt, we foreshadow it with fearful for true foreshadowing and peaceful for false foreshadowing. The event in the end is resolve in the alarmed mood.

Lastly, the girl realizes how her efforts to save the snowman are in vain and she despairs and the snowman consoles her about its imminent melting. Just before this event we foreshadow it by using a content mood for true foreshadowing or miserable mood for false foreshadowing.

\subsubsection{Why procedural music generation}

Imagine doing a similar study with classical music composition: we would have had to compose a number of different scores, record a performance of them and then insert the resulting music pieces in our game. As you can see, this would have been a time consuming and complicated effort, considering that the events that trigger the music changes aren't scripted, but triggered by the player.

By using procedurally generated music, which is fully dynamic and able to change to a different mood at any time, we took away all this overhead of prior composition. If we consider the cost in time for implementing the audio system

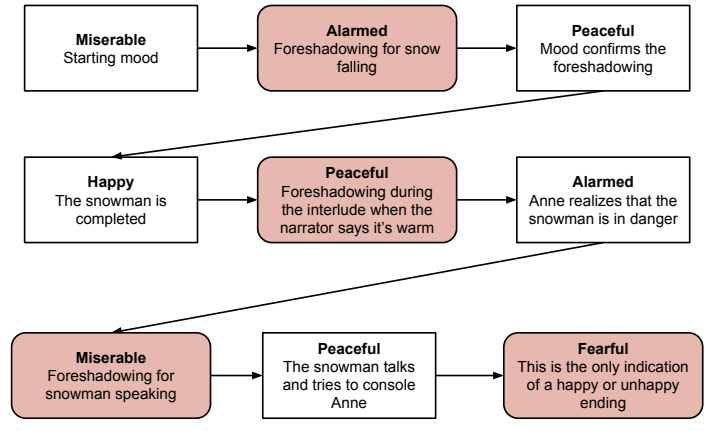

Figure 2: The emotional structure with false foreshadowing (for Group 1). In white we have the persistent moods and in red the false foreshadowing ones.

in the game engine we probably would have had less work to do considering classical composition (many tools exist, for example, for loop-based soundtracks), while we had to implement the interaction with our Pure Data generator. We still believe that the advantages of the procedural music generation approach greatly outmatch the disadvantages of using classically written music.

\subsection{Data Collection}

\subsubsection{Survey questions}

We collected more information directly from the participants by having them complete a survey after having played the game. The survey consists of the following questions:

1. "Select the three moods that you felt more while playing the game": in this question the participants could select up to three of the eight affect words describing the moods we can express with our music generator.

2. "Did you find the music consistent with the narrative?": the participant selects one of the five point Likert scales [21] (1=Strongly Disagree, $2=$ Disagree, $3=$ Neutral, $4=$ Agree, $5=$ Strongly Agree).

3. "Write the first three events that come to mind about the story": this question was designed to test the impact of music foreshadowing on the recall of story events.

4. "Rate these key events by emotional signifi-

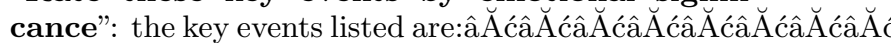

- First time seeing the snow

- Completing the snowman

- Noticing the snowman might be melting

- The snowman speaks 
These events represent the resolution of the music foreshadowing as discussed in section 3.1.4. We employ the five points Likert scale $(1=$ Very low significance, $2=$ Low significance, $3=$ Medium significance, $4=\mathrm{High}$ significance, $5=$ Very high significance).

5. "How much surprise did you feel at these key points?": the participant selects one of the five points Likert scale $(1=$ Not surprising, $2=\mathrm{A}$ little surprising, $3=$ Surprising, $4=$ Very surprising, $5=$ Extremely surprising).

6. "How much did you enjoy the game?": the participant selects one of the five points Likert scale $(1=$ Not at all, $2=$ Enjoyed a little, $3=$ Enjoyed it, $4=$ Very much enjoyed it, $5=$ Extremely enjoyed it).

7. Personal questions including demographic information (e.g., gender, age) and experience with written narratives and video games.

\section{RESULTS}

A total of thirty undergraduate and graduate students at IT University of Copenhagen in Denmark participated in the study. The number of participants for three test groups are evenly distributed, ten participants for each group.

\subsection{Music consistency with narrative}

First we applied statistical analyses to the data to test how music impact the gameplay experience. Our first hypothesis was that players will perceive a higher consistency of the music with the narrative when (true or false) foreshadowing has been employed in contrast with the static music provided for the control group. Table 1 shows the means of the participants' ratings about consistency. A difference of $22.5 \%$ was found between the two foreshadowing groups and the control group.

However, the Fisher's test for independence (see Table 2) suggests that the difference is not statistically significant. However, it is noted that the difference is non-trivial $(\mathrm{p}=0.09)$ when the comparison is made between the two groups employing foreshadowing and the control group.

We can also try grouping all the even slightly positive answers (answers 2 to 5 in the Likert scale) and test them

Table 1: Means of the music consistency ratings. Values can range from a minimum of 1 to a maximum of 5 .

\begin{tabular}{|l|lll|}
\hline & True F. & False F. & Control \\
\hline Consistency & 3.6 & 3.6 & 2.7 \\
\hline
\end{tabular}

Table 2: The $p$ values calculated with Fisher's test for the consistency ratings. (We have omitted the comparison between the true foreshadowing groups and false foreshadowing group, as the groups gave exactly the same answers.)

\begin{tabular}{|l|lll|}
\hline & All & True F./Control & Grouping answers \\
\hline$p$ & 0.552 & 0.337 & 0.089 \\
\hline
\end{tabular}

Table 3: Means of the general enjoyment ratings. Values can range from a minimum of 1 to a maximum of 5 .

\begin{tabular}{|l|lll|}
\hline & True F. & False F. & Control \\
\hline Enjoyment & 2.7 & 3.3 & 2.5 \\
\hline
\end{tabular}

Table 4: The $p$ values calculated with Fisher's test for the enjoyment ratings.

\begin{tabular}{|l|l|}
\hline Comparisons & $p$ \\
\hline All groups & 0.074 \\
True F. / False F. & 0.045 \\
True F. / Control & 0.546 \\
False F. / Control & 0.046 \\
\hline
\end{tabular}

against the strongly negative one: in this case we obtain a p-value of almost $9 \%$.

\subsection{General enjoyment of the game}

We analyzed the data to test the hypothesis that the participants in the groups that had foreshadowing music would enjoy the game more than the control group. The data is the one collected through the survey question regarding enjoyment (see section 3.2.1)

We note that the means of enjoyment ratings (see Table 3) for Group 0 and Group 1 are higher than those ratings obtained from the participants in Group 2. In fact, we have a respective difference from the control group's score of $5 \%$ and $20 \%$ for the True and False foreshadowing groups.

The Fisher's test on the data shows a promising result of $p=0.074$ (see Table 4), although not statistically significant. Interestingly, a significant difference was found in the player's enjoyment between the True foreshadowing and the False foreshadowing groups. This means that the participants experience higher enjoyment when false foreshadowing was used than when true foreshadowing was used.

\section{DISCUSSION AND FUTURE WORKS}

While research is very active in the fields of procedurally generated music, studies on musical (and narrative) foreshadowing combined with audio in games have been rarely conducted. Our study was set out to explore if mood expressive music can foreshadow game narrative events and its impact on the gameplay experience. We designed and implemented a game prototype and collected the players' psychological perception about key story events, emotional states, and the recall of story events.

We will now summarize our empirical findings to answer our research questions stated earlier (All the empirical results are described in depth in section 4) and do some final consideration of what these might mean:

Q1. In games, can procedurally generated mood expressive music serve as an indicator of narrative foreshadowing? The high value of $\mathrm{p}$ through Fisher's test (between all the three groups) that we showed in section 4.1 is due to the fact that we're testing all the 
groups together, while our hypothesis implies a dependency between the true foreshadowing group and the false foreshadowing group.

The p-value calculated between any of the foreshadowing groups against the control group, while being a better result, is not statistically significant. This is possibly due to the small amount of participants and relatively big amount of possible answers: each group has ten participants of which each can choose one out of five answers. The p-value calculated by grouping the positive answers together (see Table 2), however, shows a potential relationship between perceived music consistency and the use of foreshadowing.

Q2. Can the use of musical foreshadowing improve the player's experience? The enjoyment mean ratings of the participants (see Table 4.2) show that there is a possible difference for the False foreshadowing group. The Fisher's test (see Table 4) shows that there is a statistically significant difference between how much the true and false foreshadowing groups enjoyed the game.

Confronting the data from these groups with the control group we notice that we don't have a statistically significant difference between the true foreshadowing group and the control group. We can then infer that the use of true foreshadowing results neither positive nor negative towards the enjoyment of the story compared with a static soundtrack.

We can then conclude that probably the usage of false foreshadowing creates a more enjoyable experience than true foreshadowing. We hypothesize that this is due to false foreshadowing producing an additional element of surprise, which added more interest than the case in which foreshadowing caused the player to expect the outcome. It's worth noting that our study had only true or false foreshadowing, whereas it is possible for soundtracks to mix both true and false foreshadowing in one game. Therefore we don't suggest that composer should avoid true foreshadowing entirely, only that using false foreshadowing to subvert the players' expectations, at least occasionally, may increase the role the music plays in heightening narrative interest.

In the future, we plan to further investigate differences in instantaneous emotional reactions when using musical foreshadowing.

Finally, it would be very interesting to expand our music generator to include musical phrases and then test how the use of these impacts memory and foreshadowing (as it does for movie events in Boltz et al. study [6]).

There are a few limitations that are important to recognize in our study. First, there were a limited number of test participants. Second, the study was conducted with only one instance of one genre of game, which may not generalize to the broad space of possible game designs. The number of test participants, while not being too small for us to obtain any meaningful result, gave us some inconclusive data, especially when the answers were free text. It also provided for a more difficult analysis of the five-point Likert scale answers, since each group of ten participants could choose between five possible answers.

In hindsight, we probably should have tested the mood selector before conducting the experiment, and changed its design to make it more appealing. Another problem with it was the lack of explanation of its function, which was only provided verbally to each participant before the experiment. A tutorial or some on screen indications might have made its purpose more understandable and increased its usage.

Lastly, we should consider the limitations dictated by our game: we chose to make an adventure game because of the important narrative component of these games, but this is of course only one particular genre and we might obtain different results in a first person shooter. Further studies would be necessary to prove or disprove the transferability of our results in other game genres.

Not only through our study we have shown how through procedurally generated music we can express foreshadowing in our game, but we have identified some characteristics that distinguish true and false musical foreshadowing and found some of the limits of the influence we can exert on players through these techniques. We believe this knowledge can be useful for future researchers as well as for composers, game writers, and game designers.

\section{ACKNOWLEDGMENTS}

This research was partially supported by the Brain Korea 21 Plus Program through the National Research Foundation (NRF) funded by the Ministry of Education of Korea (10Z20130000013).

\section{REFERENCES}

[1] B.-C. Bae and R. M. Young. A use of flashback and foreshadowing for surprise arousal in narrative using a plan-based approach. In Interactive Storytelling, pages 156-167. Springer, 2008.

[2] R. Barthes. S/Z. Collins Publishers, 1974.

[3] C. D. Batson, L. L. Shaw, and K. C. Oleson. Differentiating affect, mood, and emotion: Toward functionally based conceptual distinctions. 1992.

[4] C. Beedie, P. Terry, and A. Lane. Distinctions between emotion and mood. Cognition 85 Emotion, 19(6):847-878, 2005.

[5] D. Birchfield. Generative model for the creation of musical emotion, meaning, and form. In Proceedings of the 2003 ACM SIGMM workshop on Experiential telepresence, pages 99-104. ACM, 2003.

[6] M. Boltz, M. Schulkind, and S. Kantra. Effects of background music on the remembering of filmed events. Memory \& Cognition, 19(6):593-606, 1991.

[7] C. R. Brewin. Cognitive change processes in psychotherapy. Psychological review, 96(3):379, 1989.

[8] D. Brown. Mezzo: An adaptive, real-time composition program for game soundtracks. In Proceedings of the AIIDE 2012 Workshop on Musical Metacreation, pages 68-72, 2012.

[9] S. B. Chatman. Story and discourse: Narrative structure in fiction and film. Cornell University Press, 1980. 
[10] A. J. Cohen. Music as a source of emotion in film. Music and emotion: Theory and research, pages 249-272, 2001.

[11] Double Fine Productions. Broken Age, 2014.

[12] M. Eladhari, R. Nieuwdorp, and M. Fridenfalk. The soundtrack of your mind: mind music-adaptive audio for game characters. In Proceedings of the 2006 ACM SIGCHI international conference on Advances in computer entertainment technology, page 54. ACM, 2006.

[13] G. Genette and J. E. Lewin. Narrative Discourse: An Essay in Method. Cornell University Press, 1983.

[14] P. Hayward. Terror Tracks: Music, Sound, and Horror Cinema. Equinox, 2009.

[15] A. Hitchcock. Psycho, 1960.

[16] B. Houge. Cell-based music organization in Tom Clancy's EndWar. Demo at the AIIDE 2012 Workshop on Musical Metacreation, 2012.

[17] H. Katayose, M. Imai, and S. Inokuchi. Sentiment extraction in music. In Proceedings of the 9th International Conference on Pattern Recognition, pages 1083-1087, 1988.

[18] G. Kreutz, U. Ott, D. Teichmann, P. Osawa, and D. Vaitl. Using music to induce emotions: Influences of musical preference and absorption. Psychology of Music, 36(1):101-126, 2008.

[19] R. S. Lazarus. Emotion and Adaptation. Oxford University Press, 1991.

[20] J. S. Lerner and D. Keltner. Beyond valence: Toward a model of emotion-specific influences on judgement and choice. Cognition \& Emotion, 14(4):473-493, 2000.

[21] R. Likert. A technique for the measurement of attitudes. Archives of Psychology, 1932.

[22] E. Lindström, P. N. Juslin, R. Bresin, and A. Williamon. "Expressivity comes from within your soul": A questionnaire study of music students' perspectives on expressivity. Research Studies in Music Education, 20(1):23-47, 2003.

[23] S. R. Livingstone and A. R. Brown. Dynamic response: Real-time adaptation for music emotion. In Proceedings of the 2nd Australasian Conference on Interactive Entertainment, pages 105-111, 2005.

[24] G. Lucas and S. Spielberg. Indiana Jones franchise, 1981-2008.
[25] LucasArts. The Secret of Monkey Island, 1990.

[26] LucasArts. Grim Fandango, 1998.

[27] B. A. Martin. The influence of gender on mood effects in advertising. Psychology $\&$ Marketing, 20(3):249-273, 2003.

[28] Maxis. Spore, 2008.

[29] Nintendo. The legend of zelda: Ocarina of time, 1998.

[30] F. Peinado and P. Gervás. Minstrel Reloaded: From the magic of Lisp to the formal semantics of OWL. In Technologies for Interactive Digital Storytelling and Entertainment, pages 93-97. Springer, 2006.

[31] E. M. Pryzby and A. Thomas. Audio foreshadowing in a wagering game machine. 2005.

[32] A. Rollings and E. Adams. Fundamentals of game design. New Challenges for Character-Based AI for Games. Chapter 20: Artificial Life and Puzzle Games. Prentice Hall, pages 573-590, 2006.

[33] J. A. Russell. A circumplex model of affect. Journal of Personality and Social Psychology, 39(6):1161-1178, 1980.

[34] M. Scirea. Mood dependent music generator. Advances in Computer Entertainment, pages 626-629, 2013.

[35] M. Scirea, Y.-G. Cheong, and B.-C. Bae. Mood expression in real-time computer generated music using Pure Data. In Proceedings of the 13th International Conference on Music Perception and Cognition, 2014. (To appear).

[36] N. Shaker, J. Togelius, and M. J. Nelson. Procedural Content Generation in Games: A Textbook and an Overview of Current Research. Springer, 2014. (To appear).

[37] B. D. Smith and G. E. Garnett. Improvising musical structure with hierarchical neural nets. In Proceedings of the AIIDE 2012 Workshop on Musical Metacreation, pages 63-67, 2012.

[38] Telltale Games. The Walking Dead, 2012.

[39] R. E. Thayer. The Biopsychology of Mood and Arousal. Oxford University Press, 1989.

[40] R. Wooller, A. R. Brown, E. Miranda, J. Diederich, and R. Berry. A framework for comparison of process in algorithmic music systems. In Generative Arts Practice 2005 - A Creativity $\&$ Cognition Symposium, 2005. 